\title{
Sozialbrache oder Wirtschaftsbrache?
}

Einige Anmerkungen zu neueren Er-darstellte.

scheinungen.

Ein alt-neuer Begriff macht die Runde in schweizerisch-geographischen Publikationen: Sozialbrache Alt ist der Ausdruck "Brache", jünger ist die Verwendung des in Deutschland geprägten Wortes "Sozialbrache". Mit etwa 20jähriger Verspätung findet nun die "Sozialbrache" Einzug in die schweizerische (zumindest in die deutsch-schweizerische) Geographie. Aber auch andere Disziplinen (so die Raumforschung und -planung, die Agronomie, die Forstwirtschaft, die Kulturingenieurwissenschaften; aber auch Soziologie und Oekonomie, usw.) nehmen diesen Ausdruck für sich in Anspruch.

Bevor man daran geht, einen neuen Ausdruck (gleiches gilt auch für alle Ausdrücke mit neuer Verwendung!) in eine Wissenschaftssprache aufzunehmen, ist es nur billig und recht, dass sich jeder Autor eine klare Vorstellung eines solchen Begriffes bildet (nach Begriffsumfang und Begriffsinhalt).

Für den Begriff "Sozialbrache" sind wir in der glücklichen Lage, dass dies schon einer für uns unternommen hat, nämlich der Geograf WOLFGANG HARTKE in seinem Aufsatz: Die "Sozialbrache" als Phänomen der geographischen Differenzierung der Landschaft. Dieser Aufsatz erschien 1956 in: Erdkunde X, S. 257269. Gerade rechtzeitig - wie man meinen möchte - erschien er nun ein zweitesmal 1969 im Rahmen des von W. STORKEBAUM herausgegebenen Buches: Sozialgeographie (Wissenschaftliche Buchgesellschaft, Darmstadt). HARTKE geht darin von der "überkommenen" Auffassung aus, dass die Ursache der Brachlegung hauptsächlich in einer Bevölkerungsabnahme zu suchen sei; damit zusammenhängend, ergibt sich eine direkte Verknüpfung von Siedlungswüstung mit Flurwüstung.

Dieser Annahme widersprachen aber seine Beobachtungen im südwestdeutschen Raum nach dem 2. Weltkrieg:

1. Brachphänomene trotz Bevölkerungszunahme

2. Flurwüstungen ohne Siedlungswüstungen

3. Es wurde festgestellt, dass nicht die schlechtesten Böden zuerst brach fielen, sondern dass die Qualität der Böden überhaupt kein signifikantes Kriterium dabei
Gerade diese letzte Beobachtung ist wesentlich für das Verständnis des Bracheproblems, denn sie zeigt, dass keine ökonomischen Theorien, resp. Gesetze, für deren Interpretation herangezogen werden können (va. nicht die Gesetze von Angebot und Nachfrage, auch nicht Grenznutzentheorien). Dies hat dann HARTKE dazu bewogen, für die spezielle Art von Brache den Begriff "Sozialbrache" zu postulieren.

Jedoch nicht allein der ökonomischen Nichtinterpretation wegen, sondern vielmehr weil es sich bei der genaueren Untersuchung herausstellte, wie dieses Brachproblem von gesellschaftlichen Faktoren induziert wird.

Eine notwendige Vorbedingung für das Entstehen von Sozialbrache ist ein labiles Gleichgewicht der beiden Sozialgruppen Bauern und Arbeiter, das sich im Vorhandensein von Arbeiterbauern resp. Bauernarbeitern manifestiert. Dieses labile Gleichgewicht muss dann aber immer mehr einem stabilen Ungleichgewicht weichen, und zwar zu gunsten cier Arbeiter. Bei diesem Uebergang vom Bauern zum Arbeiter gab und gibt es in der Regel eine mehr oder weniger lange Phase von Arbeiterbauerntum, wobei das bäuerliche Einkommen resp. der bäuerliche Besitz die Funktion einer Art "Sozialversicherung" übernahm, aber nur solange, als die Sozialversicherung eines Staates noch wenig entwickelt war, was in Südwestdeutschland bis etwa zum Ende des 2. Weltkrieges der Fall war.

Mit der allmählichen Wandlung der Wertvorstellungen über den Status von Bauern und Arbeitern, wodurch derjenige der Arbeiter angehoben wurde, hat die erwähnte bäuerliche "Sozialversicherung" ihre Bedeutung weitgehend verloren. Ausdruck, vielleicht aber auch gerade Ausgangspunkt dieses Prozesses sind die zahlreichen materiellen Besserstellungen der Arbeiter gegenüber den Vollbauern und erst recht gegenüber den Arbeiterbauern, so z. B. die 40 -Stundenwoche, die Pensionskassen, bezahlter Urlaub, aber auch die gewerkschaftliche Organisierung zur Durchsetzung von Lohnforderungen und dergleichen. Der frühere Arbeiterbauer findet es nicht mehr der Mühe wert, neben seiner Tätigkeit in der "Fabrik" noch sein (meist kleines) Anwesen zu bewirtschaften. 
Typisch dabei ist eben, dass die finanziellen Ueberlegungen kaum eine Rolle spielen. Durch die hohen Anforderungen des heutigen Wirtschaftslebens an physische und psychische Kräfte der Arbeiterbauern, ist dieser gezwungen sich für das eine oder andere zu entscheiden. Die Wahl fällt meist zugunsten des Arbeiterstandes aus, da damit (meist) ein höheres Prestige verbunden ist. Um sich doch noch gegen wirtschaftliche Rückschläge etwas abzusichern, wird das Land in vielen Fällen weder verkauft noch verpachtet, und so fällt es dann brach.

Zusammenfassend sieht HARTKE das Hauptkriterium für die Anwendung des Begriffes "Sozialbrache" darin, dass der bisherige Bearbeiter des Bodens auf die Dauer aus der agrarischen Sozialgruppe ausscheidet. Somit ist die Sozialbrache ein typisches Landschaftsphänomen für den Uebergang von der agrarischen zur industriellen Gesellschaft und kommt als solche nur dort vor. HARTKE ist es somit m. E. gelungen, eine klare Definition für den Begriff "Sozialbrache" zu geben, die auch von eminent praktischem Nutzen ist (gedacht sei an die Planung).

Um so mehr ist man dann erstaunt über die recht unscharfe Definition von "Brachland", die man in einer Publikation von SURBER, E., AMIET, R. und KOBERT, H. über: Das Brachlandproblem in der Schweiz (Bericht Nr. 112 der Eidg. Anstalt für das forstliche Versuchswesen, Birmensdorf 1973, 138S. ) auf Seite 9 und 10 finden kann. Es werden dort Grenzertragsbrache Strukturbrache und Sozialbrache unterschieden. Die Ursache dieser Brachlegungen wird in den sozio-ökonomischen resp. volks- und betriebswirtschaftlichen Verhältnissen gesehen, was m. E. so ziemlich auf dasselbe hinausläuft. Eine Gegenüberstellung von Wirtschaftsbrache und Sozialbrache mit ihrer ökonomischen resp. soziologischen Bedingung, analog einer Aufspaltung in Wissenschaft von der Gesellschaft (Soziologie) und eine Wissenschaft von der Wirtschaft (Oekonomie) scheint mir effizienter zu sein.

Das vorherige Staunen wandelt sich dann aber in Verstehen, denn die Autoren scheinen den richtungsweisenden Aufsatz von HARTKE nicht zu kennen (aus demFehlen im Literaturver- zeichnis zu schliessen).

Diese schweizerische Arbeit ist trotzdem

bemerkenswert, denn sie behandelt das Brach-

landproblem aus der Warte des Forstingenieurs und lehrt uns, dass das Aufforsten kein Allerweltsmittel gegen die negativen Auswirkungen der Brachlegung von Kulturland ist. Die zahlenmässigen Angaben über die Flächengrösse der Vergandung in der Schweiz sind rein hypothetisch zu nehmen und erfordern möglichst viele konkrete Fallstudien auf Gemeindeebene, so wie sie etwa E.W. ALTHER von der Kantonalen Fachstelle für Bodennutzung in Flawil (St. Gallen) für einen Teil der Gemeinde Curio im Tessin publizierte (Sozialbrache und Landschaftspflege. Die Abnahme der Kulturlandfläche von 1873 bis heute, am Beispiel der Gemeinde Curio im Malcantone, in: Schweizerische Zeitschrift für Vermessung, Kulturtechnik und Photogrammetrie, Dezember 1974, S. 109-116). ALTHER spricht zwar von Sozialbrache, nach der Definition von HARTKE scheint es mir sehr fraglich, ob es sich bei dieser Brache um Sozialbrache handelt und nicht vielmehr um Grenzertragsböden (also um Strukturbrache nach SURBER et al. , oder nach meiner Terminologie um Wirtschaftsbrache). Denn in dieser Studie wird der starke Bevölkerungsschwund von 448 Einwohnern im Jahre 1845 auf 256 im Jahre 1971 dokumentiert, v. a. infolge Abwanderung. Also geht in Curio die Flurwüstung mit der Siedlungswüstung einher (sofern nicht die Siedlungswüstung kompen, siert oder gar überkompensiert wird durch Ferienhäuser und -wohnungen).

Interessant sind aber doch die Ausführungen über die Möglichkeiten der Rückführung von Brachland in Kulturland. Es zeigte sich hier nämlich, dass trotz teilweise hundertjähriger Brache kein natürlicher Neuwald entstand. ALTHER sieht reelle Schancen für gewisse Teile des Gebietes in Rinderzucht und -mast, aber auch im Maisanbau.

Dass es dringend notwendig ist, das Brachlandproblem im Rahmen der Planung interdisziplinär auf kommunaler und regionaler Ebene anzugehen, macht dieser Aufsatz deutlich, v. a. wenn man bedenkt, dass nicht nur in Curio $87 \%$ der ehemaligen landwirtschaftlichen Nutzfläche brach liegen. 
Es ist $\mathrm{zu}$ hoffen, dass noch mehr solcher Fallstudien in Angriff genommen werden, denn nur so bekommen wir eine einigermassen gesicherte Vorstellung vom Ausmass des Brachlandproblems in der Schweiz, was eine notwendige
Voraussetzung bildet für ein optimales Planen. $\mathrm{Zu}$ wünschen wäre allerdings noch eine stärkere Berücksichtigung sozialgeographischer und gemeindesoziologischer Aspekte.

\section{H. R. Brunner}

\section{Literaturbesprechungen}

HAGGETT PETER:L'analyse spatiale en géographie humaine. Trad. de H. Fréchou. --Paris: A. Colin ( 1973 ). 390p.

"L'analyse spatiale en géöraphie humaine"est la traduction d'un ouvrage, paru en 1965 et devenu célèbre, de P.HAGGETT:"Licational Analysis in Human Geography". Le titre du livre est quelque peu trompeur: en fait, la problématique et les méthodes qu'il traite sont celles de la géographie théorique, qui est relativement récente, si l'on excepte les travaux de VON THUENEN, CHRISTALLER ou LOESCH. La géographie traditionnelle, empirique, n'y est mentionnée qu'à de rares occasions. Dans la premiere partie de l'ouvrage, "Les modeles de structure des localisations", l'auteur passe en revue une quantité d'études consacrées à la théorie de la localisation. Le plan qu'il adopte est progressif. Il part des mouvements (par exemple les flux dans une région polarisée) dont il présente les relations avec la distance (modeles gravitationnels), la surface (concept de champ) et le temps (concept de diffusion), ce qui l'amène à exposer la question des réseaux. La plupart des mouvements suivent des voies précises: voies de communication essentiellement,HAGGETT envient à présenter des recherches consacrées à la confrontation entre les réseaux tels qu'ils s'observent dans la réalité et les modeles obtenus compte tenu d'un certain nombre de contraintes logiques. L'étape sui vante l'amène naturellement à considérer les noeuds, c'est -à-dire les sommets des réseaux qui, en géographie humaine, sont surtout constitués par les unités de peuplement. La hiérarchie existant entre les noeuds est traitée dans une nouvelle étape ou il expose les travaux de CHRISTALLER sur les places centrales, de LOESCH (qui a perfectionné le système de CHRISTALLER) et deWEBER sur la localisation des industries. Mais, en considérant'l'ossature du système régional (le réseau des voies de communication et la hiérarchie des noeuds) et les mouvements qui en assurent la cohésion", on laisse de côté une grande partie de l'espace. $C^{\prime}$ est pourquoi il clot cette premiere partie par un chapitre consacré aux surfaces, chapitre dans lequel il traite des problemes de variation de densité et d'utilisation du sol.

La deuxieme partie de l'ouvrage est consacrée aux methodes. Là aussi, l'auteur utilise un plan progressif. Dans un premier chapitre sont exposées les principales méthodes statistiques de collecte des données (problernes des échantillons, des sondages, etc.). Une fois collectées, ces données doivent être traitées de telle manière que les structures spatiales qu'elles révèlent puissent faire l'objet d'une description. Cette description est le theme du chapitre suivant. P. HAGGETT y expose les techniques cartographiques qui permettent une localisation absolue, et les techniques d'analyse statistique qui permettent une localisation relative (par exemple un réseau de transport peut être défini par un indice de connexité ou par un indice de forme). Il se consacre ensuite à la définition des régions, dans un chapitre qu'il ouvre en regrettant la curieuse réserve des géographes à ce sujet, alors que, par ailleurs, les études régionales ont fait l'objet d'une multitude de publications. П $\mathrm{y}$ explique les diverses techniques conduisant 